\title{
Acral lesions in a pediatric population during the COVID-19 pandemic: a case series of 36 patients from a single hospital in Spain
}

\author{
Pau Rosés-Gibert ${ }^{1} \mathbb{D}$. Javier Gimeno Castillo ${ }^{1}$ Amaia Saenz Aguirre ${ }^{1} \cdot$ Francisco Javier De la Torre Gomar ${ }^{1}$. \\ Lucía Carnero González ${ }^{1}$ · Zuriñe Martinez de Lagrán Alvarez de Arcaya ${ }^{1} \cdot$ Ricardo Gonzalez-Perez $^{1}$
}

Received: 12 June 2020 / Accepted: 24 August 2020 / Published online: 8 September 2020

(c) Children's Hospital, Zhejiang University School of Medicine 2020

\begin{abstract}
Background To describe the cutaneous acral findings in a pediatric population noticed during this pandemic.

Methods A retrospective descriptive study was performed collecting data on 36 patients under 14 years old, presenting suspicious acral skin manifestations for coronavirus disease 2019 (COVID-19).

Results Patients were mostly male (63.8\%). The mean age was 11.11 years. $66.67 \%$ of patients showed erythematous papules, and $44.44 \%$ purpuric macules. Feet were affected in $97.22 \%$ of patients and hands in $5.55 \%$. Lesions were asymptomatic in $50 \%$ of patients. $30.55 \%$ of patients showed extracutaneous findings, preceding skin lesions in 12.62 days. Seven patients underwent specific severe acute coronavirus 2 (SARS-CoV-2) testing; all of these patients tested negative.

Conclusions The association between these symptoms and SARS-CoV-2 remains unclear. We recommend using these manifestations as a sign of SARS-CoV-2 infection in children. This could lead to the examination of asymptomatic and mildly symptomatic children so that contagions may be avoided.
\end{abstract}

Keywords Acral lesions · Acute acro-ischemia $\cdot$ Chilblain-like $\cdot$ COVID-19

\section{Introduction}

In December 2019, pneumonia cases caused by a novel coronavirus known as severe acute coronavirus 2 (SARS-CoV-2) were described initially. This disease then was named as coronavirus disease 2019 (COVID-19) [1]. COVID-19 spread quickly throughout the globe, leading to a pandemic [2], announced by March 11, 2020, by the World Health Organization. To date (August 10, 2020), Spain is the 11th most affected country in the world with 314,362 cases and 28,503 deaths reported.

We have thoroughly reviewed the literature concerning cutaneous manifestations of COVID-19. The most common cutaneous manifestations reported are an erythematous, vesicular or urticarial rash [3-6]. There have also been described cases presenting acral erythematous or purpuric lesions [7-13], which may also be common. There are few

Pau Rosés-Gibert

pau_roses@hotmail.com

1 Araba University Hospital, Francisco Leandro de Viana Street, 01009 Vitoria, Spain reports of these manifestations in the pediatric population $[9,12,13]$. At Araba University Hospital, in Vitoria-Gasteiz (North of Spain), the number of consultations for acral lesions in children greatly surpassed the usual, prompting further exploration.

\section{Methods}

\section{Study design}

We performed a retrospective descriptive study of our pediatric population (considered under 14 years old in Spain). 36 children from our area (Álava, Spain) presented to our institution with acral skin symptoms consistent with those previously described for COVID-19. The present study includes patients evaluated from April 7-27 2020. All human and animal studies were approved by an institutional review board.

Due to the pandemic status, data on all of the patients were obtained through teledermatology consultations referred from pediatricians in our area. For this reason, further investigations were limited. 


\section{Data recording methods}

Data on these 36 children were compiled and then recorded using Microsoft Excel 2010. We recorded age, sex, medical history and epidemiological environment (contact with confirmed or suspected COVID-19 patients). The pattern of cutaneous lesions (purpuric macules and erythematous papules) and their location and duration were assessed. Symptomatology related to skin manifestations (pain, pruritus or asymptomatic) and other extracutaneous manifestations suggestive of COVID-19 infection (fever, cough, dyspnea, diarrhea, myalgia, anosmia, ageusia), plus their temporary relationship with skin manifestations (antecedent, coincident, subsequent) were collected as well. Additionally, any diagnostic tests performed, treatments received, and evolution of both the skin findings and the infection were recorded.

\section{Statistical analysis}

The statistical analysis was performed using Microsoft Excel 2010. Age and duration of the lesions were quantitative variables. Arithmetic mean and median age were calculated. Other variables were qualitative or dichotomous and were summarized using percentages.

\section{Results}

Our results are summarized in Table 1 . Some clinical pictures of the lesions can be found in Figs. 1 and 2.

Of 36 patients included in our study, $23(63.89 \%)$ were male. The ages ranged from 3 to 13 years, with mean age being 11.1 years and median age 13 years. Three $(8.33 \%)$ of the patients had close contact with health workers. Twelve $(33.33 \%)$ of the patients reported close contact with individuals diagnosed with or with a clinically suspected COVID19 infection. None of the patients reported changes in medications a month before the onset of the cutaneous symptoms.

Clinical findings included erythematous papules in 24 (66.67\%) patients, similar to chilblains. Sixteen (44.44\%) patients had purpuric macules. Four $(11.11 \%)$ patients manifested both. One or more erosions were present in five (13.89\%) cases, and swelling was present in six (16.67\%) patients. Thirtyfive (97.22\%) patients showed involvement of the feet, whereas the hands were affected in two (5.55\%) patients. One (2.77\%) patient had both hands and feet affected. Lesions were bilateral in $21(58.33 \%)$ patients. In individuals with bilateral lesions, 10 (47.62\%) were symmetric, and 11 (52.38\%) were asymmetric.

In foot involvement, the dorsum was affected in 21 $(65.71 \%)$ cases. The pulps of toes were the second most frequent location, seen in $12(34.28 \%)$ patients. Heels were
Table 1 Cutaneous and extracutaneous manifestations $(n=36)$

\begin{tabular}{ll}
\hline Variables & Values, $n(\%)$ \\
\hline Morphology & \\
Erythematous papules & $24(66.67)$ \\
Purpuric macules & $16(44.44)$ \\
Both & $4(11.11)$ \\
Erosion & $5(13.8)$ \\
Swelling & $6(16.67)$ \\
Distribution & \\
Hands & $2(5.55)$ \\
Feet & $35(97.22)$ \\
Both & $1(2.78)$ \\
Laterality & \\
Unilateral & $20(41.67)$ \\
Bilateral & $21(58.33)$ \\
Symmetric & $10(47.62)$ \\
Asymmetric & $11(52.38)$ \\
Symptomatology & \\
Pruritus & $14(38.89)$ \\
Pain & $8(22.22)$ \\
Asymptomatic & $18(50.00)$ \\
Extracutaneous manifestations $(n=11)$ & \\
Frequency & $11(30.55)$ \\
Type & \\
Respiratory & $7(63.64)$ \\
Non-respiratory & $4(36.36)$ \\
Latency period (d) & 12.62 \\
Symptoms & \\
Cough & $7(63.64)$ \\
Fever & $3(27.27)$ \\
Diarrhea, nausea and vomiting & \\
\hline
\end{tabular}

affected in five (14.28\%) patients. Plantar involvement was noted in four $(11.42 \%)$ patients. Both children with hand involvement had lesions on the dorsum. None of the patients

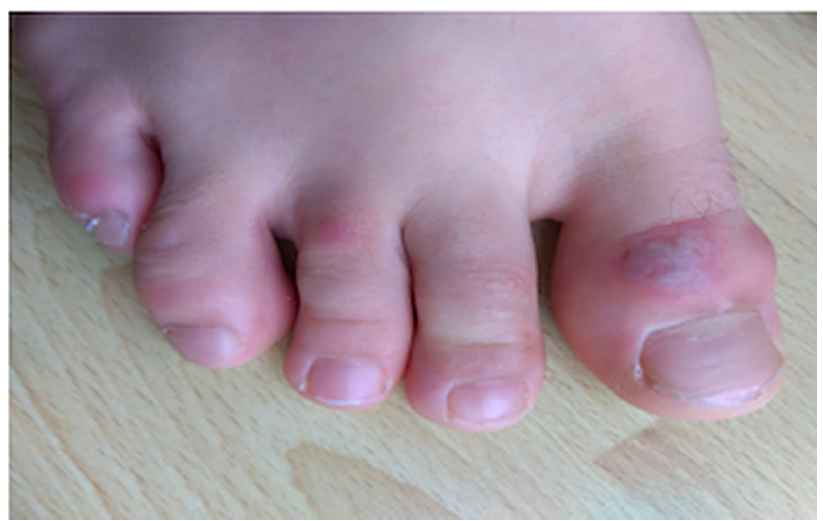

Fig. 1 Erythematous chilblain-like plaques distributed in the dorsum of the right toes 


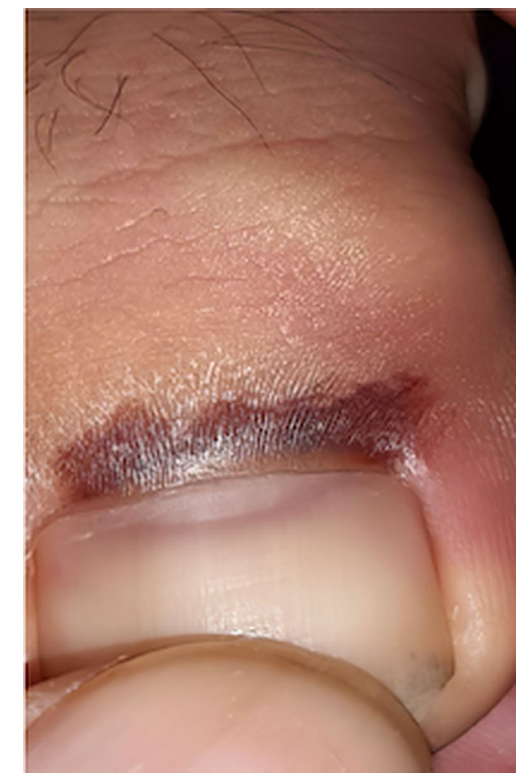

Fig. 2 Purpuric brownish macule affecting to proximal nail border of the first toe of the right foot

presented mucous involvement. Skin manifestations were asymptomatic in half $(50 \%)$ of the patients. Pruritus was the most commonly reported symptom, $(n=14,38.89 \%)$, followed by pain $(n=8,22.22 \%)$. Thus, six $(16.66 \%)$ patients utilized topical treatments, such as topical steroids or antibiotics, owing to local discomfort or ulceration.

Extracutaneous findings were seen in 11 (30.55\%) patients, of whom 7 (63.64\%) showed respiratory symptoms (cough). Fever (27.27\%) and diarrhea (27.27\%) were seen in three patients. Two (18.18\%) patients presented asthenia and myalgia. Cutaneous manifestations developed after extracutaneous symptoms in eight $(72.73 \%)$ patients with a mean latency of 12.62 days. In three $(27.27 \%)$ cases, both cutaneous and extracutaneous symptoms coincided or overlapped.

Seven patients underwent specific COVID-19 testing [all seven had polymerase chain reaction (PCR) testing, and one had serology as well (Immunochromatography test, Wondfo Biotech, Guangzhou, China)]. The mean latency regarding the performance of these tests following the onset of the symptoms was 5.14 days. All the tests were negative for SARS-CoV-2.

\section{Discussion}

During the pandemic outbreak of COVID-19, we have seen some distinctive cutaneous findings in children. These cases can present with acral erythematous papules located predominantly on the dorsa of the fingers, mimicking chilblains. Recently, we have received an unusually high number of consultations concerning purpuric macules, mostly located on the plantar side of both heels. Interestingly, they have occurred up to 25 days after the start of COVID-19-related quarantine.

The etiology of acral lesions remains unclear [14]. Some authors [15] consider that the outbreak of these lesions might be caused as a result of lifestyle changes due to the quarantine. The importance of cold weather has also been postulated to be a major trigger to these lesions [16]. Nevertheless, as a result of the concurrent outbreak of COVID-19, we consider that SARS-CoV-2 is likely to be associated. On the one hand, a microangiopathic and inflammatory process has been documented in some patients $[8,11,17]$. Magro et al. [17] reported that COVID-19 infection can cause activation of complement through the alternative and leptinmediated pathways, leading to inflammation and thrombus formation. Subsequently, Kanitakis et al. [18] gave support to this hypothesis, finding endothelialitis, microthrombi and fibrin deposits, as well as immunoglobulin (IgM, IgA) and C3 deposits, on dermal vessels when direct immunofluorescence was performed. In parallel, Colmenero et al. [19] eventually reported the presence of viral particles within endothelial cells via electron microscopy. We consider this theory might be the most accurate explanation regarding the delay of these cutaneous manifestations and its causality.

On the other hand, published articles by Landa et al. [11] and Piccolo et al. [10] propose that these lesions could represent a delayed antigen-antibody immunological reaction to SARS-CoV-2. This could explain their development in asymptomatic and mildly symptomatic patients as well as their delayed course compared to extracutaneous manifestations. In addition, negativity of PCR in nasopharyngeal swab could also be explained by this statement. Clinically, chilblain-like lesions, as well as purpuric macules, tend to be asymptomatic and to evolve with an indolent course [9, 10, 12, 13]. They usually appear in asymptomatic or paucisymptomatic patients that lack the additional criteria needed to order a COVID-19 specific test $[7,9,10]$. Histopathological findings could be helpful to reach the diagnosis. In previously reported similar cases, histopathological analysis showed superficial and deep lichenoid, perivascular and peri-eccrine infiltrate of lymphocytes, with vacuolar alteration of the basal layer and with scattered single necrotic keratinocytes [7, 9]. Red cell extravasation and dermal edema are also often described [19]. The published data about complementary blood tests reveal the lack of relevant findings [7, 9, 12, 20]. Unfortunately, we were not able to perform either a skin biopsy or blood tests for any of our patients owing to the lack of resources and the remote evaluation.

In some patients with clinical criteria for probable COVID-19 infection, PCR from a nasopharyngeal swab was performed, as well as serologic testing for antibodies in one patient. We did not obtain laboratory confirmation of COVID-19. We believe that COVID-19-negative results do not exclude this association, taking into account the low rate 
of positive PCR tests in children presenting with suggestive symptoms of SARS-CoV-2. As far as we are concerned, the swab negativity could be explained by the disappearance of detectable viral presence after a brief asymptomatic course [12]. Moreover, the sensitivity of some of the currently available serological tests is low, which supports our statement.

The management of patients presenting chilblain-like lesions and acral purpuric macules is not well established. They usually remain asymptomatic or show an indolent course; therefore, no treatment is needed $[7,9]$. Some patients from our series used topical steroids for local discomfort. Topical antibiotics also were recommended if the lesions showed any signs of necrosis or ulceration. This clinical description could pave the way for the development of deeper studies, including histopathological analysis, blood tests (including complete blood count and coagulopathy screening), and more accurate COVID-19 serologic tests. If the results validate our findings, acral cutaneous manifestations will represent a useful clue to identify COVID-19 in asymptomatic and paucisymptomatic children and will help to identify contacts and potential need for quarantine.

Acknowledgements We would like to thank all our colleagues in the Dermatology Department of Araba University Hospital for their collaboration. In addition, we would also like to thank the pediatricians from our hospital and the local primary care centers who have collaborated and helped us to perform this study.

Author contributions RGP and GCJ contributed equally to this manuscript. RGP and GCJ are the principal investigators of this manuscript. All authors contributed to the concept, design, acquisition of data, analysis and interpretation of data, drafting the article and revising it critically for important intellectual content. All authors have approved the submission of the manuscript.

Funding This research received no specific grant from any funding agency in the public, commercial or not-for-profit sectors.

\section{Compliance with ethical standards}

Ethical approval All human and animal studies were approved by the Institutional Review Board of Araba University Hospital.

Conflict of interest The authors declare that there is no conflict of interest.

\section{References}

1. Zhu N, Zhang D, Wang W, Li X, Yang B, Song J, et al. A novel coronavirus from patients with pneumonia in China, 2019. N Engl J Med. 2020;382:727-33.

2. Cucinotta D, Vanelli M. WHO declares COVID-19 a pandemic. Acta Biomed. 2020;91:157-60.

3. Recalcati S. Cutaneous manifestations in COVID-19: a first perspective. J Eur Acad Dermatol Venereol. 2020;34:e212-3.
4. Henry D, Ackerman M, Sancelme E, Finon A, Esteve E. Urticarial eruption in COVID-19 infection. J Eur Acad Dermatol Venereol. 2020;34:e244-5.

5. Su CJ, Lee CH. Viral exanthem in COVID-19, a clinical enigma with biological significance. J Eur Acad Dermatol Venereol. 2020;34:e251-2.

6. Marzano AV, Genovese G, Fabbrocini G, Pigatto P, Monfrecola G, Piraccini BM, et al. Varicella-like exanthem as a specific COVID19-associated skin manifestation: multicenter case series of 22 patients. J Am Acad Dermatol. 2020;83:280-5.

7. Kolivras A, Dehavay F, Delplace D, Feoli F, Meiers I, Milone L, et al. Coronavirus (COVID-19) infection-induced chilblains: a case report with histopathological findings. JAAD Case Rep. 2020;6:489-92.

8. Alramthan A, Aldaraji W. A case of COVID-19 presenting in clinical picture resembling chilblains disease: first report from the Middle East. Clin Exp Dermatol. 2020;45:746-8.

9. Romaní J, Baselga E, Mitjà O, Riera-Martí N, Garbayo P, Vicente A, et al. Chilblain and acral purpuric lesions in Spain during covid confinement: retrospective analysis of 12 cases. Actas Dermosifiliogr. 2020;111:426-9 (in English, Spanish).

10. Piccolo V, Neri I, Filippeschi C, Oranges T, Argenziano G, Battarra VC, et al. Chilblain-like lesions during COVID-19 epidemic: a preliminary study on 63 patients. J Eur Acad Dermatol Venereol. 2020;34:e291-3.

11. Landa N, Mendieta-eckert M, Fonda-pascual P, Aguirre T. Chilblain-like lesions on feet and hands during the COVID-19 pandemic. Int J Dermatol. 2020;59:739-43.

12. Recalcati S, Barbagallo T, Frasin LA, Prestinari F, Cogliardi A, Provero MC, et al. Acral cutaneous lesions in the time of COVID19. J Eur Acad Dermatol Venereol. 2020;34:e346-7.

13. Mazzotta F, Troccoli T. Acute acro-ischemia in the child at the time of COVID-19. Eur J Pediat Dermatol. 2020;30:71-4.

14. Docampo-Simón A, Sánchez-Pujol MJ, Juan-Carpena G, PalazónCabanes JC, Vergara-De Caso E, Berbegal L, et al. Are chilblainlike acral skin lesions really indicative of COVID-19? A prospective study and literature review. J Eur Acad Dermatology Venereol. 2020. https://doi.org/10.1111/jdv.16665.

15. Herman A, Peeters C, Verroken A, Tromme I, Tennstedt D, Marot $\mathrm{L}$, et al. Evaluation of chilblains as a manifestation of the COVID19 pandemic. JAMA Dermatology. 2020. https://doi.org/10.1001/ jamadermatol.2020.2368.

16. Joob B, Wiwanitkit V. Comment on "Chilblains-like lesions in children following suspected COVID-19 infection". Pediatr Dermatol. 2020;37:441.

17. Magro C, Mulvey JJ, Berlin D, Nuovo G, Salvatore S, Harp J, et al. Complement associated microvascular injury and thrombosis in the pathogenesis of severe COVID-19 infection: a report of five cases. Transl Res. 2020;220:1-13.

18. Kanitakis J, Lesort C, Danset M, Jullien D. Chilblain-like acral lesions during the COVID-19 pandemic ("COVID toes"): histologic, immunofluorescence and immunohistochemical study of 17 cases. J Am Acad Dermatol. 2020;83:870-5.

19. Colmenero I, Santonja C, Alonso-Riaño M, Noguera-Morel L, Hernández-Martín A, Andina D, et al. SARS-CoV-2 endothelial infection causes COVID-19 chilblains: histopathological, immunohistochemical and ultrastructural study of seven paediatric cases. Br J Dermatol. 2020. https://doi.org/10.1111/BJD.19327.

20. Roca-Ginés J, Torres-Navarro I, Sánchez-Arráez J, Abril-Pérez C, Sabalza-Baztán O, Pardo-Granell S, et al. Assessment of acute acral lesions in a case series of children and adolescents during the COVID-19 pandemic. JAMA Dermatol. 2020. https://doi. org/10.1001/jamadermatol.2020.2340.

Publisher's Note Springer Nature remains neutral with regard to jurisdictional claims in published maps and institutional affiliations. 